\title{
MORFOMETRIA DE ESPÉCIES FLORESTAIS PLANTADAS NAS CALÇADAS
}

\author{
MORPHOMETRY OF FOREST SPECIES PLANTED ON SIDEWALKS
}

Rogério Bobrowski ${ }^{1}$, Daniela Biondi²

\section{RESUMO}

A copa da árvore é a principal estrutura que influencia na oferta de benefícios ambientais, estéticos e econômicos. Entretanto, as alterações de formato podem comprometer a produção desses benefícios e potencializar riscos. O objetivo desta pesquisa foi analisar as alterações morfométricas de espécies florestais plantadas nas calçadas, notadamente em relação à rede de transmissão de energia elétrica. Os dados utilizados provêm de um inventário realizado na cidade de Curitiba em 2010, do qual foram selecionadas seis espécies com no mínimo quinze indivíduos por rua, com quatro ruas avaliadas por espécie. Foram amostradas árvores sob rede de transmissão de energia e outras longe deste tipo de estrutura. A variabilidade observada para os índices morfométricos possibilitou caracterizar a variabilidade da forma das espécies e as diferenças de conformação das copas. Não houve diferença significativa para a expressão dos índices morfométricos das árvores sob rede de transmissão de energia e árvores sem proximidade com esta estrutura, exceto para o índice formal de copa para as espécies Tipuana tipu e Lagerstroemia indica.

Palavras-chave: Poda; Forma da copa; Estrutura urbana; Arborização de ruas.

\begin{abstract}
Tree crown is the most important structure that influences on offering environmental, aesthetic and economic benefits. However, format changes may compromise the production of those benefits and enhance risks. The objective of this research was to analyze morphometric changes of forest species planted on sidewalks, mainly in relation to power line structures. Data used came from an inventory held in 2010 in the city of Curitiba from which were selected six species with at least fifteen trees by street, with four streets evaluated by species. Trees were sampled under power lines and away from this kind of structure. The variability observed for morphometric indexes made it possible to characterize the variability of species form and differences of crown formats. There was no significant difference for the expression of morphometric indexes of trees under power lines and trees away from this structure except for crown form index of species Tipuana tipu and Lagerstroemia indica.
\end{abstract}

Keywords: Pruning; Tree crown format; Urban structure; Tree-lined street.

Recebido em 24.10.2016 e aceito em 11.01.2017

1 Engenheiro Florestal. Doutor. Professor Adjunto da Universidade Estadual do Centro-Oeste. Irati/PR. E-mail: rogerio@irati.unicentro.br

2 Engenheira Florestal. Doutora. Professora Titular da Universidade Federal do Paraná. Curitiba/PR. E-mail: dbiondi@ufpr.br 


\section{INTRODUÇÃO}

A copa das árvores afeta a produtividade primária do ecossistema que constituem, regulando fatores como temperatura, umidade, vento e iluminação, pelo processo de fotossíntese e densidade de conformação da folhagem. Por isso, esta estrutura das árvores tem sido um dos objetos de maior atenção de políticas públicas emergentes e de discussões científicas relacionadas à gestão urbana e em especial a gestão da arborização urbana, para a qual se direcionam esforços que possibilitem o incremento da superfície recoberta por copas nas cidades (KENNEY; VAN WASSANAER; SATEL, 2011).

Por ser a copa da árvore o objeto das práticas de poda e maiores intervenções, derivadas das condições de plantio (principalmente o espaçamento) e das características ecológicas da espécie, presume-se que por meio da intensidade de intervenção se possa alterar a arquitetura característica, afetando o efeito estético que se busca com o plantio ordenado de árvores (BOBROWSKI; BIONDI, 2012; BOBROWSKI; LIMA NETO; BIONDI, 2013). De acordo com Hasenauer (1997) o crescimento ótimo das árvores é possível quando estas não são afetadas por árvores competidoras vizinhas ou quando não são afetadas por qualquer tipo de tratamento ao longo de suas vidas.

Para Durlo, Sutili e Denardi (2004), procedimentos silviculturais como a determinação do espaço de crescimento (concorrência máxima) e as avaliações de estabilidade, vitalidade e produtividade das árvores, especialmente quando não se conhece a idade das plantas, são dependentes de informações como a largura, a altura e a área de copa, das proporções entre esses fatores e das modificações sofridas por ações de manejo pretéritas. Estas observações foram reforçadas por Roman, Bressan e Durlo (2009) quando afirmaram que o conhecimento da morfometria, das relações morfométricas e da dinâmica da forma das árvores são fatores importantes para intervenções silviculturais e utilização de espécies nativas em reflorestamentos.

Estas relações morfométricas das espécies florestais podem ser descritas por índices como proporção de copa, grau de esbeltez, índice de saliência, índice de abrangência e formal de copa, os quais expressam relações dimensionais entre o diâmetro à altura do peito (DAP), largura de copa, altura de copa e altura total da árvore (DURLO; DENARDI, 1998).

De acordo com Bobrowski (2015), a proporção de copa em árvores urbanas pode ser um indicativo da qualidade de oferta de benefícios em virtude da quantidade de massa verde foliar, o grau de esbeltez pode ser utilizado para priorizar ações de avaliação da suscetibilidade de árvores à queda, o índice de saliência pode auxiliar no planejamento do espaçamento mais adequado para cada espécie para máximo crescimento e oferta de benefícios, o índice de abrangência pode ser utilizado como um indicativo para o planejamento da substituição 
gradativa das árvores e o índice formal de copa pode ser utilizado para expressar numericamente a característica estética da forma de copa das árvores e suas alterações.

Além destes índices, Kontogianni, Tsitsoni e Goudelis (2011) utilizaram o índice de assimetria da copa e o grau de esbeltez para expressar a correlação das características dimensionais da árvore com a sua estabilidade, baseado no comportamento silvicultural de quatro espécies florestais, ao estudar a arborização de ruas da cidade de Thessaloniki, na Grécia.

Entretanto, as técnicas de avaliação da morfometria e das relações morfométricas, com estudos e aplicações no manejo de florestas plantadas e nativas, mediante análise de árvores individuais, associadas na floresta ou em sistemas agroflorestais (HASENAUER, 1997; DURLO; DENARDI, 1998; DURLO; SUTILI; DENARDI, 2004; ROMAN; BRESSAN; DURLO, 2009), não são de uso comum na silvicultura urbana. Porém, isto poderia auxiliar no conhecimento das relações interdimensionais, do comportamento individual e da interação entre as árvores nas ruas, bem como auxiliar a adoção de melhores critérios de planejamento da implantação de espécies em uso ou que serão incorporadas na arborização, além do estabelecimento de limites aceitáveis de intervenção por podas.

Neste sentido, Bobrowski, Lima Neto e Biondi (2013) estudaram características morfométricas de Tipuana tipu (tipuana) na arborização de ruas da cidade de Curitiba, Paraná, e suas modificações em função da localização das árvores, se sob rede de distribuição de energia elétrica ou livre desta ou se crescendo em ambientes abertos. Neste trabalho constataram que a arquitetura típica da espécie e algumas variáveis morfométricas são alteradas significativamente quando em condições de plantio em calçadas, em relação àquelas conduzidas em áreas com maior espaçamento (praças, residências, entre outras).

Como base nestas informações os objetivos desta pesquisa foram analisar a variabilidade morfometrica de espécies florestais plantadas na arborização de ruas de Curitiba, Paraná, e as alterações morfométricas em condições de plantio sob a rede de transmissão de energia elétrica e na ausência desta.

\section{MATERIAL E MÉTODOS}

Para este estudo foram selecionadas espécies encontradas nas unidades amostrais do inventário da arborização de ruas realizado por Bobrowski (2011), na cidade de Curitiba, Estado do Paraná. Esta cidade está localizada na região Sul do Brasil e na porção leste do Estado, entre as coordenadas $25^{\circ} 25^{\prime \prime} 48^{\prime \prime S}$ e $49^{\circ} 16^{\prime \prime} 15^{\prime \prime}$. 
Para a seleção das espécies adotou-se o critério de que elas deveriam ter no mínimo 15 indivíduos por rua avaliada, distribuídos de forma contínua, um ao lado do outro e em quatro ruas diferentes, não necessariamente na mesma unidade amostral do inventário. Cada rua constituiu uma unidade de tratamento distinta, devido às características diferentes de largura da pista de rolamento, largura da calçada e largura do recuo frontal das residências.

Este critério foi adotado para que se reduzisse a influência por competição de espaço com espécies de arquitetura de copa diferentes ou pela competição derivada de espécie/árvore não regularmente plantada e que pudesse estar a uma distância inadequada da espécie objeto desta avaliação, por conta do não planejamento do espaçamento de implantação da árvore.

Em cada grupo de 15 indivíduos selecionados procurou-se amostrar árvores dos dois lados de cada rua, ou seja, em calçada com rede de distribuição de energia elétrica e em calçada sem a presença deste tipo de infraestrutura. Em algumas ruas, no trecho de quadra amostrado, não havia rede de distribuição de energia, não sendo possível fazer a coleta para ambas as situações. O número de indivíduos amostrados sob fiação e na ausência desta não foi exatamente igual para cada espécie, em virtude das restrições impostas para a pesquisa e das características dos plantios existentes.

Em virtude da existência de plantios irregulares em todas as unidades amostrais que serviram de base para a coleta de dados, o número de espécies e de árvores que atendessem às restrições metodológicas impostas foi reduzido. Desta forma, as espécies selecionadas foram as indicadas na Figura 1: Cassia leptophylla Vogel (falso-barbatimão), Handroanthus albus (Cham.) Mattos (ipê-amarelo-graúdo), Handroanthus chrysotrichus (Mart. ex A.DC.) Mattos (ipê-amarelo-miúdo), Lagerstroemia indica L. (extremosa), Parapiptadenia rigida (Benth.) Brenan (angico) e Tipuana tipu (Benth.) Kuntze (tipuana).

Para cada árvore amostrada foram obtidas as seguintes informações: altura total $(\mathrm{m})$, altura de copa $(m)$, circunferência à altura do peito $(m)$ e diâmetro de copa $(m)$. A partir destas variáveis foram calculados cinco índices morfométricos (proporção de copa, grau de esbeltez, índice de saliência, índice de abrangência e formal de copa) que expressam relações intradimensionais de fácil obtenção prática, de acordo com as descrições de Durlo e Denardi (1998); Roman, Bressan e Durlo (2009) e Bobrowski, Lima Neto e Biondi (2013). Os índices utilizados são aqueles indicados na Figura 2.

Para o índice de formal de copa (FC) os dados foram divididos em tipos de conformação, a fim de caracterizar melhor a dimensão e expressão da copa das espécies. As classes utilizadas foram: Classe 1 (FC < 0,25), Classe 2 (FC 0,25 0,9), Classe 2 (FC 0,9 1,1), Classe $2(F C 1,1+4,0)$ e Classe $5(F C>4,0)$. Cada classe de formal de copa foi ilustrada como uma figura geométrica da copa, dentre colunar, elíptica e arredondada, para demonstrar graficamente as alterações de forma observadas. 


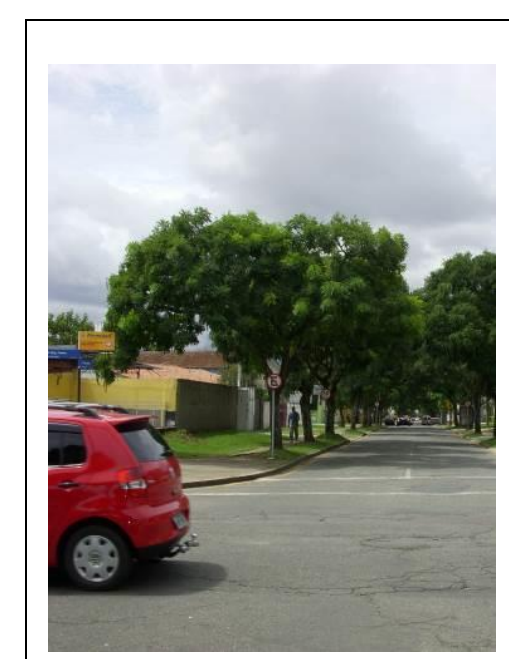

Cassia leptophylla

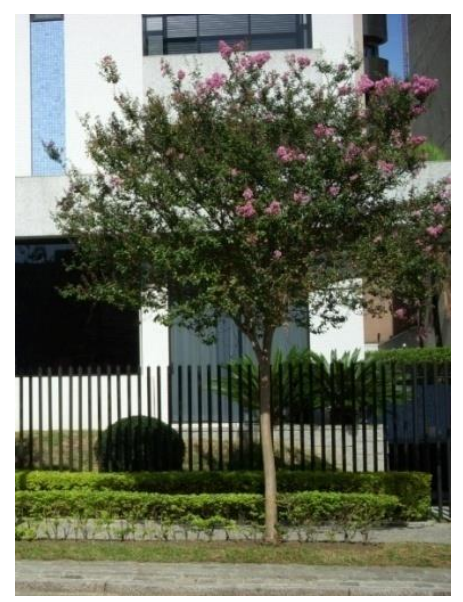

Lagerstroemia indica

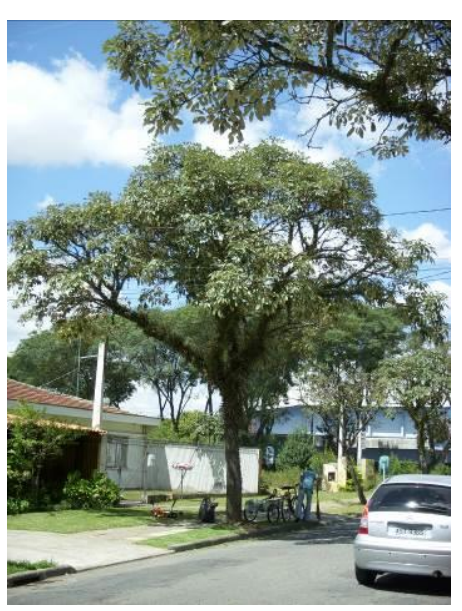

Handroanthus albus

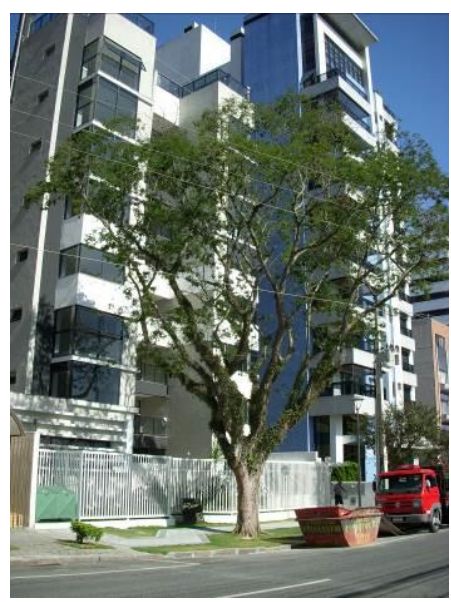

Parapiptadenia rigida

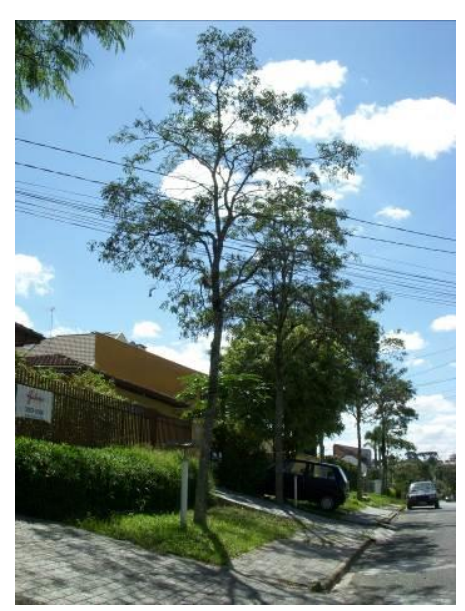

Handroanthus chrysotrichus

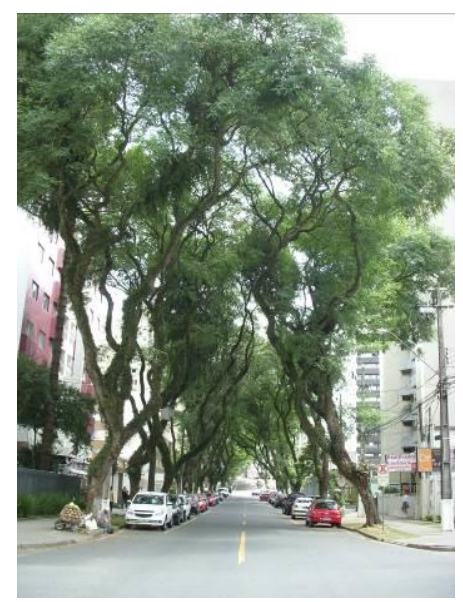

Tipuana tipu

Figura 1. Espécies florestais utilizadas para a análise morfométrica Figure 1. Forest species used for morphometric analysis

Com os dados morfométricos obtidos foi efetuada análise comparativa, por espécie, entre os dados das árvores sob rede de transmissão de energia e daquelas em calçada sem este tipo de estrutura. As análises comparativas foram realizadas com o auxílio do software Statgraphics Centurion XV utilizando o teste $t$ ao nível de $5 \%$ de probabilidade e assumindo uma amostragem aleatória irrestrita na coleta dos dados. 


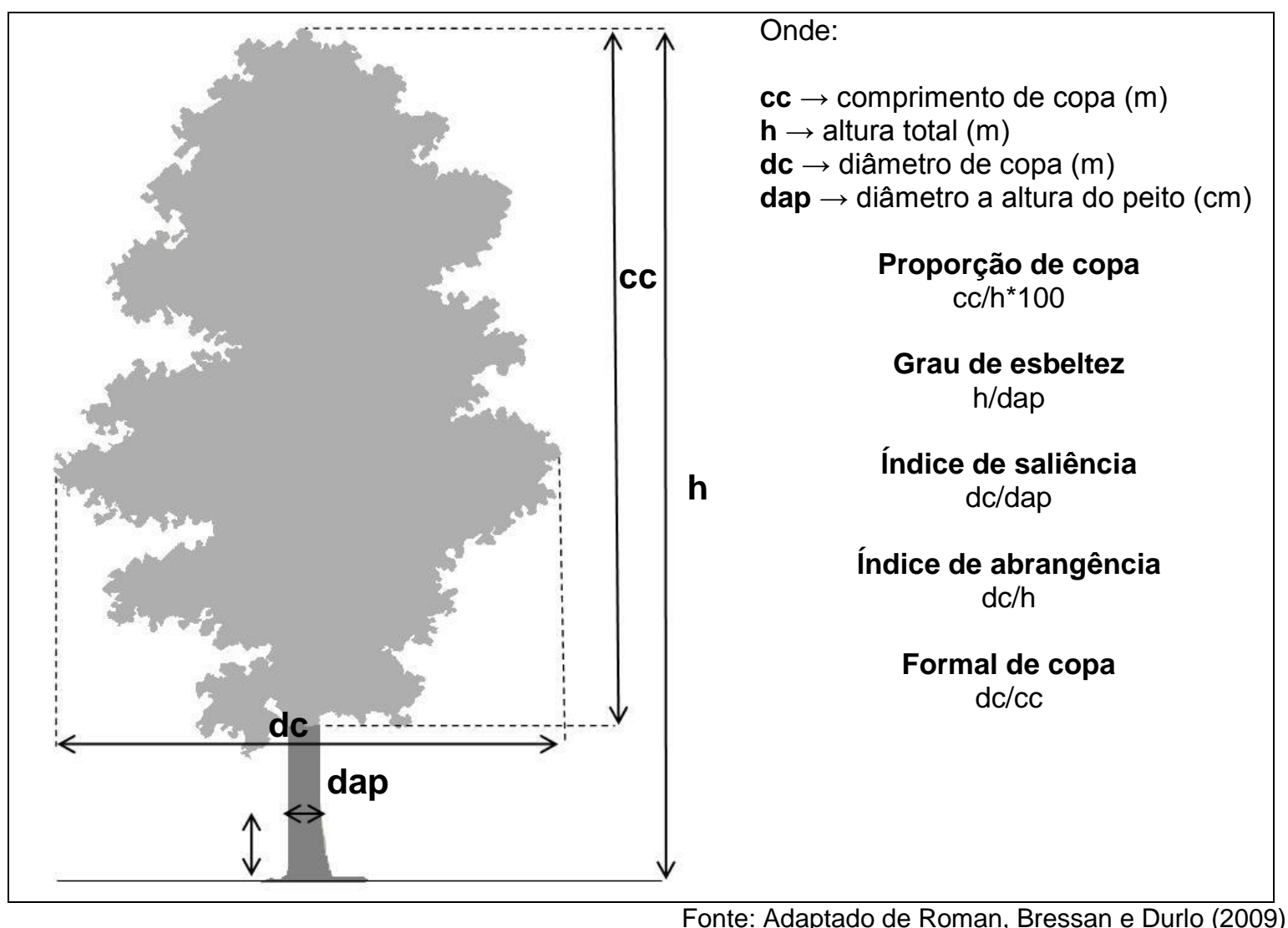

Figura 2. Índices morfométricos utilizados para análise das espécies florestais plantadas nas calçadas

Figure 2. Morphometric indexes used for the analysis of forest species planted on sidewalks

\section{RESULTADOS E DISCUSSÃO}

A variabilidade das medidas observadas para as variáveis dendrométricas das espécies florestais estudadas (Tabela 1), indicada pelo coeficiente de variação, corresponde a uma condição específica da arborização de ruas. Além das características intrínsecas das espécies, a variação nas condições ambientais de crescimento (compactação do solo e nutrição, por exemplo) e a variação das dimensões morfológicas das árvores, promovidas pelas práticas de manejo e por atos de vandalismo tendem a condicionar a obtenção de coeficientes de variação muito maiores que aqueles observados em condições experimentais ideais, que deveriam estar aproximados ou abaixo de $10 \%$. 
Tabela 1. Variabilidade das variáveis dendrométricas obtidas para a estimativa dos índices morfométricos por espécie

Table 1. Variability of dendrometric variables obtained to the estimative of morphometric indexes by species

\begin{tabular}{|c|c|c|c|c|c|c|c|c|c|c|}
\hline \multirow{2}{*}{ Variáveis } & \multicolumn{5}{|c|}{ Parapiptadenia rigida } & \multicolumn{5}{|c|}{ Tipuana tipu } \\
\hline & $\mathrm{N}$ & MIN & MÉD & MÁX & CV\% & N & MIN & MÉD & MÁX & CV\% \\
\hline Itura Copa (m) & 7 & 5,00 & 90 & 7,00 & 10,91 & 59 & 3,50 & 5,73 & 9,00 & 6,63 \\
\hline tura Total ( & & 1,00 & 17,47 & 22,50 & 20,08 & 59 & 9,00 & 15,73 & 23,50 & 18,44 \\
\hline ר) & & 0,32 & 55 & 0,81 & 22,86 & 39 & 0,32 & 57 & 1,02 & 24,66 \\
\hline |âr & & 6,40 & 13,71 & 18,45 & 18,80 & 59 & 4,80 & 14,70 & 25,25 & 27,09 \\
\hline \multirow{2}{*}{ ariáveis } & \multicolumn{5}{|c|}{ Lagerstroemia indica } & \multicolumn{5}{|c|}{ Cassia leptophylla } \\
\hline & $\mathrm{N}$ & MIN & MÉD & MÁX & CV\% & $\mathrm{N}$ & MIN & MÉD & MÁX & V\% \\
\hline Iture & , & 1,50 & 0 & 4,50 & 25,33 & 53 & 2,20 & 97 & 5.50 & 7,72 \\
\hline Itura & & 2,50 & 6,50 & 11,00 & 26,57 & 53 & 6,50 & 9,47 & 13,00 & 17,02 \\
\hline & & 09 & & 0,29 & 25,07 & 53 & 0,26 & 36 & 0,50 &, 14 \\
\hline D C & & 2,85 & 5,15 & 8,55 & 23,98 & 53 & 6,10 & 9,50 & 13,90 & 17,32 \\
\hline \multirow{2}{*}{ Variáveis } & \multicolumn{5}{|c|}{ Handroanthus albus } & \multicolumn{5}{|c|}{ Handroanthus chrysotrichus } \\
\hline & $\mathrm{N}$ & MIN & MÉD & MÁX & $\mathrm{CV} \%$ & N & MIN & MÉD & MÁX & CV\% \\
\hline & & 2,00 & & 5,50 & 23,49 & 59 & 1,00 & 3,49 & 7, & 0,72 \\
\hline Altura & 57 & 4,50 & 7,59 & 14,00 & 31,75 & 59 & 4,50 & 8,20 & 13,50 & 28,66 \\
\hline & & 0,13 & 0,25 & 0,41 & 26,73 & 59 & 0,08 & 0,16 & 0,25 & 26,26 \\
\hline 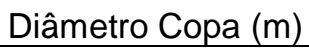 & 1 & 3,30 & 6,45 & 11,50 & 29,47 & 59 & 2,70 & 5,14 & 9,50 & 24,07 \\
\hline
\end{tabular}

Legenda: Número total de árvores (N), valores mínimos (MIN), médios (MÉD), máximos (MÁX) e coeficiente de variação (CV\%)

Entretanto, experimentos na arborização urbana apontam a ocorrência de valores fora deste limite ideal (SMILEY; KANE, 2006; BOBROWSKI; LIMA NETO; BIONDI, 2013). Para 12 espécies florestais destacadas em seu estudo, Bobrowski (2011) encontrou valores de coeficiente de variação para o DAP $(\mathrm{m})$ variando entre $30,25 \%$ e $56,87 \%$, para a altura $(\mathrm{m})$ entre $37,09 \%$ e $143,84 \%$ e para a área de copa $\left(\mathrm{m}^{2}\right)$ entre 50,85\% e 458,08\%. Faria, Souza e Miranda (2014), ao analisarem a arborização da cidade de Ituporanga, Goiás, observaram valores de coeficiente de variação entre 3,39 e 70,74\% para o diâmetro da base e entre 7,00 e $46,83 \%$ para a altura dos vegetais lenhosos amostrados.

$\mathrm{Na}$ Tabela 2 constata-se que os menores valores máximos de proporção de copa foram observados para espécies de grande porte (Parapiptadenia rigida e Tipuana tipu), ao passo que, os maiores valores máximos foram observados para espécies de pequeno porte (Lagerstroemia indica e Handroanthus chrysotrichus). Isto pode ser explicado pelo fato de que para as espécies de grande porte a prefeitura municipal costuma realizar podas de elevação, repetidas vezes, a fim de reduzir ao máximo os galhos que possam entrar em conflito com a rede de distribuição de energia elétrica, bem como para reduzir os conflitos que podem ser gerados por galhos que se projetam sobre a pista de rolamento das ruas e avenidas. Disto decorre, comumente, o que se observa em ruas e avenidas arborizadas com espécies de 
grande porte adultas: um corredor de galhos e troncos, altos, recoberto por um manto verde de folhas.

Tabela 2. Variabilidade dos índices morfométricos obtidos para cada espécie amostrada Table 2. Variability of morphometric indexes obtained for each species sampled

\begin{tabular}{|c|c|c|c|c|c|c|c|c|c|c|}
\hline \multirow{2}{*}{ Índice Morfométrico } & \multicolumn{5}{|c|}{ Parapiptadenia rigida } & \multicolumn{5}{|c|}{ Tipuana tipu } \\
\hline & $\mathrm{N}$ & MIN & MÉD & MÁX & $\mathrm{CV}^{\mathrm{c}}$ & v & 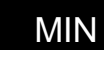 & MÉD & MÁX & CV\% \\
\hline \multirow{5}{*}{$\begin{array}{l}\text { Proporção Copa } \\
\text { Grau de Esbeltez } \\
\text { Índice de Saliência } \\
\text { Índice de Abrangência } \\
\text { Formal de Copa }\end{array}$} & & 23,26 & 34,97 & 50,00 & 19,93 & 9 & $2 \pi, V_{0}$ & דם, וש & 50,00 & 10, \\
\hline & & & 32,14 & & 15,99 & 59 & 18,85 & 28,15 & 43,98 & $9,($ \\
\hline & & 15,59 & ,43 & 37,02 & 19,74 & 59 & 12,16 & 25,57 & 32,15 & 12,73 \\
\hline & & 0,46 & 0,80 & 1,15 & 21,38 & 9 & 0,44 & 0,94 & 1,58 & 2,64 \\
\hline & & 1,07 & 2,33 & 3,17 & 17,68 & 9 & 0,96 & 2,56 & 3,80 & 9,45 \\
\hline \multirow{2}{*}{ Índice Morfométrico } & \multicolumn{5}{|c|}{ Lagerstroemia indica } & \multicolumn{5}{|c|}{ Cassia leptophylla } \\
\hline & N & MIN & MÉD & MÁX & 0 & $\mathrm{~N}$ & MIN & MÉD & MÁX & CV\% \\
\hline \multirow{5}{*}{$\begin{array}{l}\text { Proporção Copa } \\
\text { Grau de Esbeltez } \\
\text { Índice de Saliência } \\
\text { Índice de Abrangência } \\
\text { Formal de Copa }\end{array}$} & 0 & 2 & 4 & 33 & 14 & 53 & & 24 & 5 & ,70 \\
\hline & 0 & 11,06 & 35,61 & 63,81 & 30,48 & 53 & 17,16 & 26,76 & 34,56 & 14,51 \\
\hline & 0 & 88 & 28,21 & 48,08 & 27,74 & 53 & ,42 & 26,80 & 33,27 & 13,49 \\
\hline & 0 & 0,37 & 84 & 1,87 & 33,43 & 53 & 0,64 & 1,02 & 1,55 & 17,88 \\
\hline & 0 & 0,74 & 2,02 & 4,10 & 34,44 & 53 & 1,53 & 2,43 & 3,66 & 17,18 \\
\hline \multirow{2}{*}{ Índice Morfométrico } & \multicolumn{5}{|c|}{ Handroanthus albus } & \multicolumn{5}{|c|}{ Handroanthus chrysotrichus } \\
\hline & $\mathrm{N}$ & MIN & MÉD & MÁX & Cl & $\mathrm{N}$ & MIN & MÉD & MÁX & CV\% \\
\hline Pro & 57 & 29,63 & 46,16 & 70,00 & 21,69 & 59 & 14,29 & 44,12 & 72,73 & 27,66 \\
\hline & 57 & 19,39 & 30,92 & 49,21 & 21,76 & 59 & 28,05 & 53,59 & 89,15 & 27,52 \\
\hline & 57 & 19,17 & 26,00 & 32,55 & 13,24 & 59 & 22,23 & 33,43 & 49,48 & 17,62 \\
\hline minare & 57 & 0,5 & $0,8 /$ & 1 & 20, & 59 & 0,36 & 0,66 & 1,32 & 30,46 \\
\hline Formal d & 57 & 1,00 & 1,95 & 3,03 & 24,92 & 59 & 0,85 & 1,58 & 4,05 & 35,04 \\
\hline
\end{tabular}

Legenda: Número total de árvores $(\mathrm{N})$, valores mínimos (MIN), médios (MÉD), máximos (MÁX) e coeficiente de variação (CV\%)

Maiores proporções de copa para as árvores de uma determinada rua ou avenida pode ser um fator importante do ponto de vista ambiental, social, econômico e estético, pois a copa das árvores é o provedor essencial destes benefícios (BOBROWSKI; BIONDI, 2012), os quais são almejados com a implantação da arborização de ruas. Do ponto de vista ambiental, a maior proporção de copa pode ser um indicativo de melhor regulação do microclima de uma rua ou avenida, pois maior é a camada de folhas sombreando e transpirando. A barreira foliar mais espessa reduz a penetração (atenuação) da radiação solar através das copas, o que resulta na redução do aquecimento do solo, da emissão de radiação de onda longa e, por consequência, do aquecimento do ar entre o solo e o manto de copa e a redução da sensação de estresse por calor (ALI-TOUDERT; MAYER, 2006; ALI-TOUDERT; MAYER, 2007). Entretanto, a regulação de fatores microclimáticos que influenciam o conforto térmico depende das características de arquitetura de copa das espécies utilizadas, da regularidade de composição do plantio, do espaçamento adotado, da idade das árvores, da altura total, da 
densidade de copa característica e da deciduidade ou não da espécie (ABREU; LABAKI, 2010; MARTINI; BIONDI; BATISTA, 2013; MARTINI et al., 2013).

Com relação ao grau de esbeltez, o menor e o maior valor foram encontrados para $L$. indica, sendo respectivamente iguais a 11,06 e 63,81 (Tabela 2) e relacionados aos maiores coeficientes de variação observados. Essa grande variabilidade do índice pode ser explicada pela suscetibilidade da espécie a sofrer intervenções por poda e consequentes deformações na copa (BOBROWSKI, 2011), com mais intensidade que as demais espécies. Devido ao fato dos indivíduos de $L$. indica possuírem altura média em torno de 6,50 m e, por conta disso, estarem localizados entre os limites da rede de telecomunicação e baixa tensão (inferior) com a rede de média tensão (superior), mais frequentes e intensas tendem a ser as intervenções por poda a fim de eliminar os conflitos gerados com as redes aéreas.

Para Wessolly (1996), Slodicak e Novak (2006), Kontogianni, Tsitsoni e Goudelis (2011) o grau de esbeltez pode ser utilizado para avaliar a suscetibilidade das árvores à queda ocasionada pela força exercida pelo vento. Para alguns autores (WESSOLLY, 1996; DURLO; DENARDI, 1998; SLODICAK; NOVAK, 2006; ROMAN; BRESSAN; DURLO, 2009) à medida que aumenta o valor do grau de esbeltez mais instável se torna a árvore, o que pode aumentar o risco de ruptura e queda, pois a altura total tende a ser, proporcionalmente, maior que o DAP.

Entretanto, Wessolly (1996) ressalta que para se reconhecer problemas com estabilidade nas árvores deve-se considerar a altura, o grau de esbeltez e a forma da copa. Da mesma forma, há que se considerar as características anatômicas da espécie, as propriedades mecânicas, as forças envolvidas, a altura total, o diâmetro, as condições de crescimento, dentre outros fatores, porque pode haver correlação positiva ou negativa entre a esbeltez e a densidade da madeira, por exemplo, o que pode afetar diferentemente a probabilidade de ruptura da árvore (AIBA; NAKASHIZUKA, 2009).

Os valores de grau de esbeltez observados para algumas espécies nesta pesquisa são menos expressivos que aqueles obtidos para espécies em florestas naturais, onde a competição por espaço e recursos é maior e, devido a isso, há maior investimento do crescimento em altura do que em diâmetro. Para Cordia trichotoma (Vell.) Arráb. ex Steud. (louro-pardo) foram observados valores entre 47,80 e 130,00 (ROMAN; BRESSAN; DURLO, 2009), para Cabralea canjarana (Vell.) Mart (canjarana) foram observados valores entre 29,80 e 173,00 (DURLO; DENARDI, 1998).

Para as espécies analisadas encontrou-se o menor valor de índice de saliência para Tipuana tipu $(12,16)$ e o maior valor para Handroanthus chrysotrichus $(49,48)$, sendo que para a média dos valores deste índice foi observada diferença significativa entre os valores de Lagerstroemia indica e Handroanthus chrysotrichus ( $p$-valor=0,000), entre Lagerstroemia indica e Tipuana tipu ( $p$-valor=0,018), entre Lagerstroemia indica e Parapiptadenia rigida ( $p$ - 
valor $=0,025$ ) e entre Handroanthus chrysotrichus e todas as espécies comparadas ( $p$ valor $=0,00)$.

O que pode explicar a diferença existente entre os valores do índice de saliência de Handroanthus chrysotrichus em relação às demais espécies é o modelo arquitetural da espécie, do tipo ortotrópico simpodial, associado ao porte com pequena projeção de copa, pouco densa e que ocupa relativamente pouco espaço na calçada. Isso permite que a espécie possa projetar sua copa muitas vezes mais, em relação a um determinado DAP, quando comparada com as demais espécies analisadas. Para as outras espécies verifica-se um comportamento relativamente igual para os valores do índice, o que reflete um comportamento dimensional assemelhado, em termos relativos como expresso pelo índice, apesar da diferença entre o porte considerado para as mesmas. Entretanto, com relação aos menores valores do índice de saliência, como observado para Tipuana tipu, ainda não se tem conhecimento sobre qual seria o valor limitante para cada espécie a partir do qual um DAP máximo aceitável estaria relacionado a danos significativos nas calçadas por crescimento de raízes de sustentação ou transtornos e riscos pela queda de galhos de grande porte.

A diferença observada entre os valores do índice para Lagerstroemia indica, Tipuana tipu e Parapiptadenia rigida pode ser devido às diferenças entre as formas de suas copas, considerando as características descritas por Biondi e Althaus (2005). L. indica é descrita como uma espécie com copa arredondada e não difere quanto ao valor do índice de saliência de outras duas espécies com copa descrita como arredondada, Handroanthus albus e Cassia leptophylla. Para as espécies Tipuana tipu e Parapiptadenia rigida, apesar de possuírem copa arredondada/umbeliforme (BIONDI; ALTHAUS, 2005) podem ter tido a expressão de amplitude do diâmetro de copa mascarada pelas condições de tratamento dadas pelas práticas de poda ou por competição pelo entrelaçamento de copas, pois são espécies de grande porte que tendem a ser conduzidas para se reduzir os conflitos com a rede de distribuição de energia elétrica e demais estruturas urbanas e construções. Esse fato pode ser melhor compreendido quando se observa que a média dos valores deste índice para Tipuana tipu e Parapiptadenia rigida foram os menores.

Os valores do índice de abrangência variaram entre 0,36 para Handroanthus chrysotrichus e 1,87 para Lagerstroemia indica. Para estas mesmas espécies foram observados os maiores valores de coeficiente de variação (Tabela 2).

Para árvores com índice de abrangência > 1,5 a projeção da copa é muito maior que a altura total, o que pode causar problemas com queda de galhos avantajados em tamanho, devido ao peso originado, ou maior suscetibilidade da árvore à queda, devido a intervenções inadequadas que promovem o desequilíbrio na copa e comprometem a distribuição de carga. 
A capacidade e a possibilidade que a espécie tem para desenvolver a copa lateralmente, em detrimento à altura total ou altura da copa, pode ser resultado das características de arquitetura de copa e das práticas de manejo adotadas sobre as árvores. Quanto à arquitetura de copa, assemelha-se ao modelo plagiotrópico quando tende a apresentar um crescimento maior no eixo horizontal em relação ao eixo vertical da copa. Entretanto, essa tendência pode ser manipulada por meio de podas que reduzam a altura total da árvore favorecendo o seu desenvolvimento lateral. Com tendência a assumir esta característica comportamental de arquitetura plagiotrópica (alto valor para a relação diâmetro de copa/altura de copa) estão as espécies Tipuana tipu e Cassia leptophylla, as quais apresentaram os maiores valores médios para o formal de copa, sendo respectivamente iguais a 2,56 e 2,43 (Tabela 2).

Assumindo-se as classes de valores de formal de copa indicados na Figura 3 verificase que há variabilidade na forma assumida pelas copas das árvores, entre as diferentes espécies e mesmo para uma única espécie considerada. Para todas as espécies foram observados valores médios do formal de copa correspondentes à conformação de copa do tipo elíptica horizontal.

Para Parapiptadenia rigida (angico), Tipuana tipu (tipuana) e Handroanthus albus (ipêamarelo-graúdo) a conformação da copa variou de arredondada a elíptica horizontal, para Lagerstroemia indica (extremosa) e Handroanthus chrysotrichus (ipê-amarelo-miúdo) variou de elíptica vertical a colunar horizontal e para Cassia leptophylla (falso-barbatimão) apenas como elíptica horizontal. Para as espécies Tipuana tipu e Cassia leptohylla há descrição em literatura de que suas copas são do tipo elíptica (BIONDI; ALTHAUS, 2005), mas pela análise do índice formal de copa (Tabela 2) a elipsóide formada tende a ser no sentido horizontal. Para Lagerstroemia indica afirma-se que a copa seja do tipo globosa ou arredondada (BIONDI; ALTHAUS, 2005), porém, em conjunto com Handroanthus chrysotrichus, foram as espécies que apresentaram maior variabilidade na conformação da copa, associadas aos maiores coeficientes de variação para o índice formal de copa. Essa variabilidade se deve às práticas de manejo adotadas, as quais visam a redução da copa, principalmente para árvores de pequeno porte plantadas sob rede de transmissão de energia, pois a altura média observada para estas espécies foi igual a 6,50 m e 8,20 m (Tabela 1). 


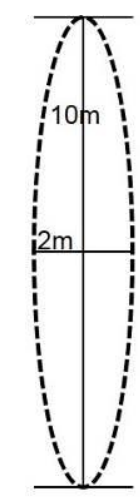

Tipo 1

Colunar Vertical FC $<0,25$

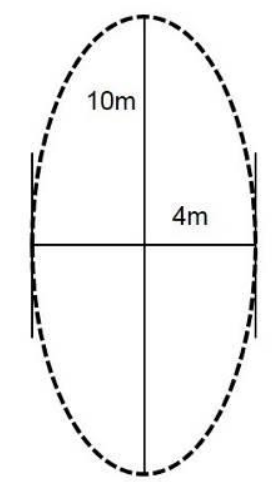

\section{Tipo 2}

Elíptica Vertical FC $0,25+0,9$

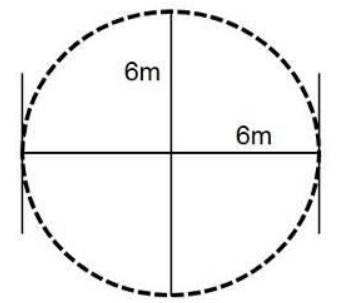

Tipo 3

Arredondada FC $0,9+1,1$

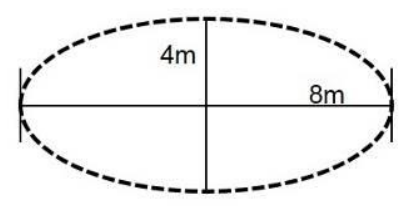

Tipo 4

Elíptica Horizontal

FC $1,1+4,0$

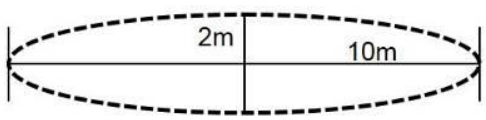

Tipo 5

Colunar Horizontal

$\mathrm{FC}>4,0$

Figura 3. Tipos de conformação da copa e respectivas classes de formal de copa (FC)

Figure 3. Kinds of crown shape and respective classes of crown form index (FC)

Os resultados das análises das árvores sob rede de transmissão e daquelas localizadas em calçadas sem esta estrutura estão apresentados nas Tabelas 3. Para estes dados foi realizada análise comparativa dos intervalos de confiança, por índice morfométrico e por espécie, da qual segue resultado na Tabela 4.

Mediante análise comparativa não foram constatadas diferenças significativas ( $p$-valor $>0,05)$ entre os valores dos índices das árvores sob rede de transmissão de energia e em calçada sem rede, exceto para o índice formal de copa para as espécies T. tipu e L. indica. Para a primeira espécie a média dos valores do índice, para as árvores em calçada sem rede de transmissão $(2,69)$, foi maior que daquelas sob rede de transmissão $(2,39)$, com uma diferença de $11,15 \%$ (Tabela 3). Por outro lado, para a segunda espécie a média dos valores das árvores sob rede foi maior $(2,33)$ que daquelas em calçada sem esta estrutura $(1,90)$, com uma diferença de $18,45 \%$. 
Tabela 3. Variabilidade dos índices morfométricos para árvores sob rede de transmissão de energia (Sob rede) e em calçada sem esta estrutura (Sem rede), por espécie

Table 3. Variability of morphometric indexes for trees under powerline (Sob rede) and in sidewalks without this structure (Sem rede), by species

\begin{tabular}{|c|c|c|c|c|c|c|c|c|c|c|c|c|}
\hline \multirow{3}{*}{ Índice Morfométrico } & \multicolumn{6}{|c|}{ Parapiptadenia rigida } & \multicolumn{6}{|c|}{ Tipuana tipu } \\
\hline & \multicolumn{3}{|c|}{ Sob rede } & \multicolumn{3}{|c|}{ Sem rede } & \multicolumn{3}{|c|}{ Sob rede } & \multicolumn{3}{|c|}{ Sem rede } \\
\hline & $\mathrm{N}$ & MÉD & $\mathrm{CV} \%$ & $\mathrm{~N}$ & MÉD & $\mathrm{CV} \%$ & $\mathrm{~N}$ & MÉD & CV\% & $\mathrm{N}$ & MÉD & CV\% \\
\hline Proporç & 23 & 33,31 & 14,13 & 34 & 36,09 & 21,79 & 27 & 38,26 & 13,01 & 32 & 36,01 & 17,97 \\
\hline Grau de Esbeltez & 23 & 31,69 & 18,05 & 34 & 32,44 & 14,18 & 27 & 29,03 & 19,72 & 32 & 27,40 & 17,59 \\
\hline Índice & 23 & 24,51 & 14,09 & 34 & 26,05 & 21,88 & 27 & 25,54 & 15,22 & 32 & 25,58 & 9,93 \\
\hline Índice de Abrangência & 23 & 0,79 & 16,59 & 34 & 0,82 & 23,45 & 27 & 0,91 & 25,96 & 32 & 0,96 & 19,03 \\
\hline Formal $d$ & 23 & 2,39 & 15,08 & 34 & 2,29 & 18,94 & 27 & 2,39 & 21,70 & 32 & 2,69 & 15,72 \\
\hline \multirow{3}{*}{ Índice Morfométrico } & \multicolumn{6}{|c|}{ Lagerstroemia indica } & \multicolumn{6}{|c|}{ Cassia leptophylla } \\
\hline & \multicolumn{3}{|c|}{ Sob rede } & \multicolumn{3}{|c|}{ Sem rede } & \multicolumn{3}{|c|}{ Sob rede } & \multicolumn{3}{|c|}{ Sem rede } \\
\hline & $\mathrm{N}$ & MÉD & CV\% & $\mathrm{N}$ & MÉD & $\mathrm{CV} \%$ & $\mathrm{~N}$ & MÉD & CV\% & $\mathrm{N}$ & MÉD & CV\% \\
\hline Proporção Copa & 17 & $40,<4$ & 33,91 & 43 & 41,03 & 22,83 & 33 & 43,07 & 16,26 & 20 & 40,86 & 9,84 \\
\hline Grau de Esbeltez & 17 & 33,36 & 27,49 & 43 & 36,50 & 30,70 & 33 & 25,97 & 14,74 & 20 & 28,04 & 12,54 \\
\hline Índice de Saliência & 17 & 29,58 & 30,74 & 43 & 27,66 & 25,64 & 33 & 26,49 & 13,17 & 20 & 27,29 & 13,47 \\
\hline Índice de Abrang & 17 & 0,90 & 31,18 & 43 & 0,81 & 33,44 & 33 & 1,04 & 18,42 & 20 & 0,98 & 15,83 \\
\hline Formal de Copa & 17 & 2,33 & 34,39 & 43 & 1,90 & 31,61 & 3 & 2,44 & 18,13 & 20 & 2,42 & 14,90 \\
\hline \multirow{3}{*}{ Índice Morfométrico } & \multicolumn{6}{|c|}{ Handroanthus albus } & \multicolumn{6}{|c|}{ Handroanthus chrysotrichus } \\
\hline & \multicolumn{3}{|c|}{ Sob rede } & \multicolumn{3}{|c|}{ Sem rede } & \multicolumn{3}{|c|}{ Sob rede } & \multicolumn{3}{|c|}{ Sem rede } \\
\hline & $\mathrm{N}$ & MÉD & $\mathrm{CV} \%$ & $\mathrm{~N}$ & MÉD & $\mathrm{CV} \%$ & $\mathrm{~N}$ & MÉD & CV\% & $\mathrm{N}$ & MÉD & CV\% \\
\hline Proporção Copa & 19 & 45,13 & 20,72 & 38 & 46,67 & 21,76 & 29 & 41,55 & 32,01 & 30 & 46,59 & 21,92 \\
\hline Grau de Esbeltez & 19 & 31,13 & 22,05 & 38 & 30,82 & 21,31 & 29 & 57,21 & 28,64 & 30 & 50,09 & 23,28 \\
\hline Índice de Saliência & 19 & 25,55 & 15,42 & 38 & 26,23 & 9,56 & 29 & 34,10 & 19,29 & 30 & 32,79 & 15,06 \\
\hline Índice de Abrangência & 19 & 0,85 & 21,39 & 38 & 0,88 & 20,11 & 29 & 0,63 & 29,13 & 30 & 0,69 & 30,19 \\
\hline Form & 19 & 1,98 & 31,22 & 38 & 1,94 & 20,34 & 29 & 1,64 & 38,30 & 30 & 1,52 & 29,59 \\
\hline
\end{tabular}

Para Tipuana tipu, a distinção observada pode ser decorrente das limitações e redução da realização de poda de elevação sob rede de distribuição de energia elétrica, a qual deve atender às distâncias de segurança dos cabos energizados definidos em regulamentações técnicas do setor de energia elétrica e ser realizada por equipe especializada para operar com redes energizadas. Por outro lado, em condições sem rede de transmissão fica-se livre para elevar a copa até os limites supostamente necessários para a espécie de grande porte objeto da prática de poda.

Para Lagerstroemia indica a condição de maior valor médio do índice formal de copa sob rede de distribuição de energia é compreensível pelo fato de que a espécie pode ter sofrido mais interferências na altura da copa, por podas consecutivas, a fim de evitar conflitos rotineiros na rede, já que em média a espécie possui altura total igual a 6,50 m (Tabela 1). 
Tabela 4. Análise comparativa entre os valores dos índices morfométricos de proporção de copa (PC), grau de esbeltez (GE), índice de saliência (IS), índice de abrangência (IA) e forma de copa (FC), por espécie

Table 4. Comparative analysis among morphometric indexes of crown ratio (PC), slenderness coefficient (GE), salience index (IS), range index (IA) and crown form (FC), by species

\begin{tabular}{lccccc} 
Espécie & $\mathbf{P C}$ & $\mathbf{G E}$ & $\mathbf{I S}$ & $\mathbf{I A}$ & $\mathbf{F C}$ \\
P. rigida & $\mathrm{t}=1,496$ & $\mathrm{t}=0,527$ & $\mathrm{t}=1,135$ & $\mathrm{t}=0,615$ & $\mathrm{t}=-0,862$ \\
& $p$-valor $(0,140)$ & $p$-valor $(0,600)$ & $p$-valor $(0,261)$ & $p$-valor $(0,541)$ & $p$-valor $(0,393)$ \\
\hline T. tipu & $\mathrm{t}=-1,452$ & $\mathrm{t}=-1,167$ & $\mathrm{t}=0,059$ & $\mathrm{t}=0,818$ & $\mathrm{t}=2,402$ \\
& $p$-valor $(0,152)$ & $p$-valor $(0,248)$ & $p$-valor $(0,953)$ & $p$-valor $(0,418)$ & $p$-valor $(\mathbf{0}, \mathbf{0 1 9})$ \\
\hline \multirow{2}{*}{ L. indica } & $\mathrm{t}=1,039$ & $\mathrm{t}=1,001$ & $\mathrm{t}=-0,858$ & $\mathrm{t}=-1,202$ & $\mathrm{t}=-2,201$ \\
& $p$-valor $(0,303)$ & $p$-valor $(0,317)$ & $p$-valor $(0,397)$ & $p$-valor $(0,234)$ & $p$-valor $(\mathbf{0}, 032)$ \\
\hline \multirow{2}{*}{ C. leptophylla } & $\mathrm{t}=-1,266$ & $\mathrm{t}=1,923$ & $\mathrm{t}=0,777$ & $\mathrm{t}=-1,045$ & $\mathrm{t}=-0,211$ \\
& $p$-valor $(0,211)$ & $p$-valor $(0,061)$ & $p$-valor $(0,440)$ & $p$-valor $(0,301)$ & $p$-valor $(0,834)$ \\
\hline \multirow{2}{*}{ H. albus } & $\mathrm{t}=0,543$ & $\mathrm{t}=-0,159$ & $\mathrm{t}=0,693$ & $\mathrm{t}=0,648$ & $\mathrm{t}=-0,375$ \\
& $p$-valor $(0,589)$ & $p$-valor $(0,874)$ & $p$-valor $(0,491)$ & $p$-valor $(0,519)$ & $p$-valor $(0,709)$ \\
\hline \multirow{2}{*}{ H. chrysotrichus } & $\mathrm{t}=1,609$ & $\mathrm{t}=-1,894$ & $\mathrm{t}=-0,852$ & $\mathrm{t}=1,194$ & $\mathrm{t}=-0,846$ \\
& $p$-valor $(0,113)$ & $p$-valor $(0,063)$ & $p$-valor $(0,398)$ & $p$-valor $(0,238)$ & $p$-valor $(0,401)$ \\
\hline
\end{tabular}

\section{CONCLUSÕES}

A variabilidade dimensional expressa pelos índices morfométricos possibilitou 0 reconhecimento de padrões de conformação das copas, tanto sob rede de transmissão de energia quanto em calçada sem este tipo de estrutura. Isto pode derivar de características das espécies, de práticas de manejo e de atos de vandalismo.

Não foi constatada diferença significativa entre as características dimensionais das árvores crescendo em calçada sob rede de transmissão de energia e calçada sem esse tipo de estrutura, exceto para o índice formal de copa para a espécies T. tipu e L. indica. Para estas espécies, as alterações significativas observadas derivam apenas das práticas de poda realizadas.

Os índices morfométricos mostram-se aplicáveis como ferramenta auxiliar do manejo e planejamento da arborização de ruas, por expressarem conformações e alterações das características naturais de crescimento das espécies.

\section{REFERÊNCIAS}

ABREU, L. V.; LABAKI, L. C. Conforto térmico propiciado por algumas espécies arbóreas: avaliação do raio de influência através de diferentes índices de conforto. Ambiente Construído, Porto Alegre, v.10, n.4, p.103-117, 2010.

AIBA, M.; NAKASHIZUKA, T. Architectural differences associated with adult stature and wood density in 30 temperate tree species. Functional Ecology, Londres, v.23, n.2, p.265-273, 2009.

ALI-TOUDERT, F.; MAYER, H. Numerical study on the effects of aspect ratio and orientation of an urban street canyon on outdoor thermal comfort in hot and dry climate. Building and Environment, Amsterdam, v.41, n.2, p.94-108, 2006. 
ALI-TOUDERT, F.; MAYER, H. Effects of asymmetry, galleries, overhanging façades and vegetation on thermal comfort in urban street canyons. Solar Energy, Amsterdam, v.81, n.6, p.742-754, 2007.

BIONDI, D.; ALTHAUS, M. Árvores de rua de Curitiba: cultivo e manejo. Curitiba: FUPEF, 2005.

BOBROWSKI, R. Estrutura e dinâmica da arborização de ruas de Curitiba, Paraná, no período 1984 - 2010. 144 f. Dissertação (Mestrado em Engenharia Florestal) - Setor de Ciências Agrárias, Universidade Federal do Paraná, Curitiba, 2011.

BOBROWSKI, R.; BIONDI, D. Distribuição e dinâmica da área de copa na arborização de ruas de Curitiba, Paraná, Brasil, no período de 1984-2010. Árvore, Viçosa, v.36, n.4, p.625-635, 2012.

BOBROWSKI, R.; LIMA NETO, E. M.; BIONDI, D. Alterações na arquitetura típica de Tipuana tipu (Benth.) O. Kuntze na arborização de ruas de Curitiba, Paraná. Ciência Florestal, Santa Maria, v.23, n.3, p.281-289, 2013.

BOBROWSKI, R. A floresta urbana e a arborização de ruas. In: BIONDI, D. Floresta urbana. Curitiba: O Autor, 2015.p-81-107.

DURLO, M. A.; DENARDI, L. Morfometria de Cabralea canjarana, em mata secundária nativa do Rio Grande do Sul. Ciência Florestal, Santa Maria, v.8, n.1, p. 55-66, 1998.

DURLO, M. A.; SUTILI, F. J.; DENARDI, L. Modelagem da copa de Cedrela fissilis Vellozo. Ciência Florestal, Santa Maria, v.14, n.2, p.79-89, 2004.

FARIA, R. F.; SOUSA, V. R.; MIRANDA, S. C. Arborização urbana da cidade de Itapuranga, Goiás. Revista da Sociedade Brasileira de Arborização Urbana, Piracicaba, v.9, n.2, p. 101117, 2014.

HASENAUER, H. Dimensional relationships of open-grown trees in Austria. Forest Ecology and Management, Amsterdam, v.96, n.3, p.197-206, 1997.

KENNEY, W. A.; VAN WASSENAER, P. J. E.; SATEL, A. L. Criteria and indicators for strategic urban forest planning and management. Arboriculture \& Urban Forestry, Champaign, v.17, n.3, p.108-117, 2011.

KONTOGIANNI, A.; TSITSONI, T.; GOUDELIS, G. An index based on silvicultural knowledge for tree stability assessment and improved ecological function in urban ecosystems. Ecological Engineering, Amsterdam, v.37, n.6, p.914-919, 2011.

MARTINI, A.; BIONDI, D.; BATISTA, A. C. Variação diária e estacional do microclima urbano em ruas arborizadas de Curitiba-PR. Floresta e Ambiente, Seropédica, v.20, n.4, p. 460-469, 2013.

MARTINI, A.; BIONDI, D.; BATISTA, A. C.; ZAMPRONI, K. A periodicidade diária do índice de conforto térmico na arborização de ruas de Curitiba-PR. Scientia Plena, Aracajú, v.9, n.5, p.19, 2013. 
ROMAN, M.; BRESSAN, D. A.; DURLO, M. A. Variáveis morfométricas e relações interdimensionais para Cordia trichotoma (Vell.) Arráb. ex Steud. Ciência Florestal, Santa Maria, v.19, n.4, p.473-480, 2009.

SLODICAK, M.; NOVAK, J. Silvicultural measures to increase the mechanical stability of pure secondary Norway spruce stands before conversion. Forest Ecology and Management, Amsterdam, v.224, n.3, p.252-257, 2006.

SMILEY, E. T.; KANE, B. The effect of pruning type on wind loading of Acer rubrum. Arboriculture and Urban Forestry, Champaign, v.32, n.1, p.33-40, 2006.

WESSOLLY, L. Stability of Trees: Explanation of the Tipping Process. Stadt und Grun, Berlin, n. 4, p. 268-272, 1996. Disponível em: <http://www2.treeconsult.org/images/pdf/article4.pdf>. Acesso em: 25/06/2013. 